\title{
Ellipsometry study of two-dimensional phase transitions
}

\author{
Th. Rasing, H. Hsiung, ${ }^{*}$ and Y. R. Shen \\ Department of Physics, University of California, Berkeley, California 94720 \\ and Center For Advanced Materials, Lawrence Berkeley Laboratory, Berkeley, California 94720 \\ M. W. Kim \\ Exxon Research and Engineering Company, Annandale, New Jersey 08801
}

(Received 28 December 1987)

\begin{abstract}
The structure of a monolayer of pentadecanoic acid at a water-air interface has been studied through its gas, liquid, and condensed phases by use of a very sensitive ellipsometry technique. The results clearly show the presence of two coexistence regions on the isotherm and a nonuniform film at full coverage.
\end{abstract}

Monolayers of long-chain molecules spread at a waterair interface are of great interest because of their relevance to molecular electronics, ${ }^{1}$ biological membranes, ${ }^{2}$ and because of the fundamental interest in twodimensional phase transitions. ${ }^{3}$ At low densities they often exhibit a two-dimensional gas (G) behavior, ${ }^{4}$ whereas at higher densities, transitions to the so-called liquid-expanded (LE), liquid-condensed (LC), and possible solid (S) phases occur. ${ }^{3}$

The nature of the different phase transitions has long been controversial due to the limiting experimental probes (surface-tension and potential measurements). Recently, progress has been made through the development of new techniques, probing the microscopic features of the transitions, and by improvement of the older techniques. The LE-LC transition has been shown to be of first order. ${ }^{5}$ By using optical second-harmonic generation, ${ }^{6}$ the reorientation of the molecules upon compression has been shown experimentally, and recently the ordering of the hydrocarbon chains has been measured using infrared-visible sumfrequency generation. ${ }^{7}$

In this paper we will show how we can use a very sensitive linear ellipsometry technique to obtain additional information about the structure of a monolayer of pentadecanoic acid [PDA, $\mathrm{CH}_{3}\left(\mathrm{CH}_{2}\right)_{13} \mathrm{COOH}$ in its $\mathrm{G}, \mathrm{LE}$, and LC phases. The results show that both the $G$ and LE and the LE and LC phases are separated by coexistence regions on the isotherm, consistent with a first-order character of both transitions. The LE phase is shown to be homogeneous whereas at full monolayer coverage the film appears not be be uniform but rather consists of domains. The results do not support the distinction between a LC and a solid phase.

A monolayer at an interface between media 1 (air) and 2 (water) can be considered as an infinitely thin dielectric sheet represented by an induced polarization.

$$
\mathbf{P}_{s}=\chi_{s} \mathbf{E},
$$

where $\mathbf{E}$ is the electric field at the interface $(z=0)$ and $\chi_{s}$ is the surface susceptibility $\chi_{s}=\chi_{s} \delta(z)$; for simplicity here taken as a scalar, i.e., the monolayer is assumed to be isotropic. With the light incident from medium 1, the $s$ - and $p$-polarized fields radiated by this dipole layer back into medium 1 are given by

$$
\begin{aligned}
& E_{s}^{1}=\frac{4 \pi i k_{0}^{2}}{k_{1 z}+k_{2 z}} P_{s, y}, \\
& E_{p}^{1}=\frac{4 \pi i k_{1} / \epsilon_{1}}{k_{1 z} / \epsilon_{1}+k_{2 z} / \epsilon_{2}}\left(\frac{k_{2 z}}{\epsilon_{2}} P_{s, x}+k_{x} P_{s, z}\right),
\end{aligned}
$$

where $k_{0}=\omega / c$. The total reflected field will be the sum of $E^{1}$ and the bulk reflection. From this the reflection coefficients for both $s$ and $p$ waves can be derived as

$$
\begin{aligned}
& r_{s}=r_{s}^{0}\left(1+\frac{8 \pi i k_{0}^{2} k_{1 z}}{k_{1 z}^{2}-k_{2 z}^{2}} \chi_{s}\right), \\
& r_{p}=r_{p}^{0}\left(1+\frac{8 \pi i k_{1 z} / \epsilon_{1}\left[k_{x}^{2}-\left(k_{2 z} / \epsilon_{2}\right)^{2}\right] \chi_{s}}{\left(k_{1 x} / \epsilon_{1}\right)^{2}-\left(k_{2 z} / \epsilon_{2}\right)^{2}}\right),
\end{aligned}
$$

where $r_{s}^{0}(p)$ represents the coefficients for the bare interface. The expression for the phase retardation is then obtained as

$$
\begin{aligned}
\Delta \phi & =\phi_{s}-\phi_{p} \\
& =\chi_{s} 8 \pi k_{1 z}\left[\frac{k_{0}^{2}}{k_{1 z}^{2}-k_{2 z}^{2}}-\frac{1}{\epsilon_{1}}\left(\frac{k_{x}^{2}-\left(k_{2 z} / \epsilon_{2}\right)^{2}}{\left(k_{1 z} / \epsilon_{1}\right)^{2}-\left(k_{2 z} / \epsilon_{2}\right)^{2}}\right)\right] .
\end{aligned}
$$

From Eq. (4) it follows that $\Delta \phi$ is linearly proportional to the surface susceptibility $\chi_{s}$. For a monomolecular layer and in the absence of local-field effects $\chi_{s}$ can be related to the molecular polarizability $\alpha^{(1)}$ by

$$
\chi_{s}=N_{s}\left\langle\alpha^{(1)}\right\rangle
$$

where $N_{s}$ is the surface density of molecules and the angular brackets indicate an orientational average. Thus, $\Delta \phi$ is linearly proportional to $N_{s}$.

As an alternative approach, one can consider a monolayer as a dielectric film of thickness $d$ and bulk dielectric constant $\epsilon_{m}=1+4 \pi \chi_{m} .{ }^{8}$ In that case, the reflection coefficients have the form

$$
r=\frac{r_{1 m}+r_{m 2} e^{2 i k_{m z} d}}{1+r_{1 m} r_{m 2} e^{2 i k_{m x} d}},
$$


where $r_{i j}$ is the reflection coefficient between media $i$ and $j$ and the subscript $m$ indicates the monolayer. One can easily show that in the limit $d \rightarrow 0$ but $d \chi_{m}=\chi_{s}=$ finite; this leads to exactly the same result as Eq. (4).

Monolayers of PDA were prepared by spreading a solution of PDA in petroleum ether on a thoroughly cleaned water surface $(p H=2)$. The trough was made out of glass and the edges were coated with paraffin. The surface density of molecules was controlled by a movable Teflon barrier and the surface tension $\pi$ was measured by a Wilhelmy plate. ${ }^{9}$ For the ellipsometry measurements, we used a weak He-Ne laser at $632.8 \mathrm{~nm}$ incident on the water surface at $\theta_{i} \approx 75^{\circ}$. The phase shift $\Delta \phi$ was measured by a standard high-resolution ellipsometry technique. ${ }^{10}$ The accuracy in $\Delta \phi$ was $-10^{-4} \mathrm{rad}$.

Figure 1 shows some typical ellipsometry data obtained at three different parts of the phase diagram of a monolayer of PDA on water at $21^{\circ} \mathrm{C}$. Figure 2 summarizes the results of both surface pressure and ellipsometry measurements. For $0.13<N_{s}<1.9 \times 10^{14} \mathrm{~cm}^{-2}$, the horizontal plateau in the $\pi-N_{s}$ curve indicates the coexistence region between the $G$ and LE phases. The corresponding measured $\Delta \phi$ appears to fluctuate very strongly [see Fig. 1 (a)]. Plotted in Fig 2(b) are the peak-to-peak amplitudes of the fluctuating ellipsometry signal at a given density (with the present sensitivity of $\sim 10^{-4}$ rad the signal in the pure $G$ phase for $N_{s}<0.13 \times 10^{14} \mathrm{~cm}^{-2}$ was too small to be detected). For $1.9<N_{s}<2.5 \times 10^{14} \mathrm{~cm}^{-2}$, the monolayer is in the pure LE phase, characterized by an increasing $\pi$ and a stable $\Delta \phi$ [see Fig. 1(b)] that is linearly increasing with $N_{s}$, the latter in accordance with Eqs. (4) and (5). For $N_{s} \geq 2.5 \times 10^{14} \mathrm{~cm}^{-2}$, the LE-LC transition point as

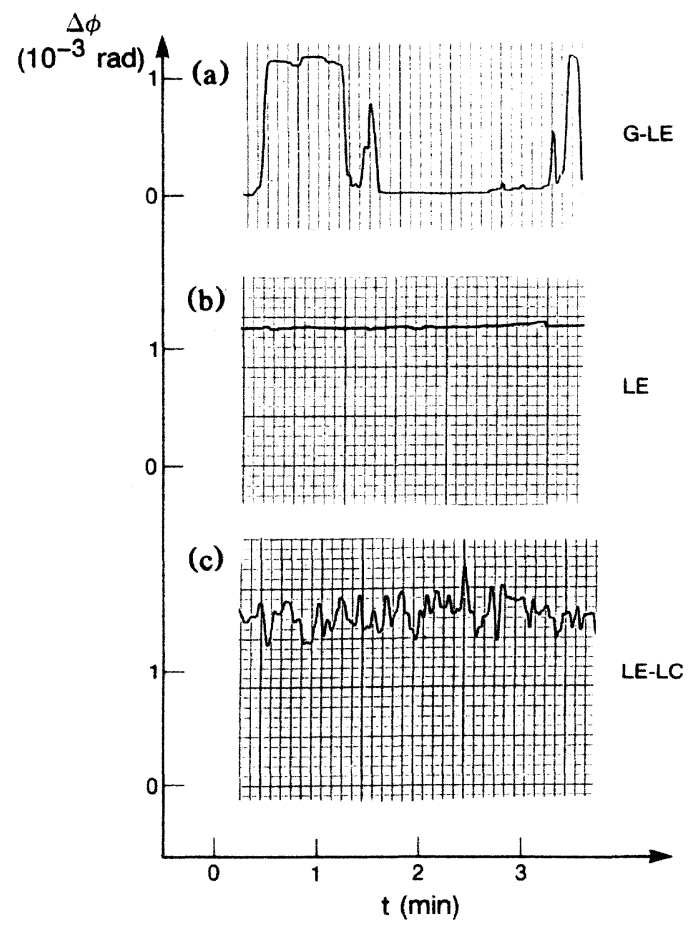

FIG. 1. Some typical ellipsometry data for three different parts of the phase diagram of a monolayer of PDA on water.

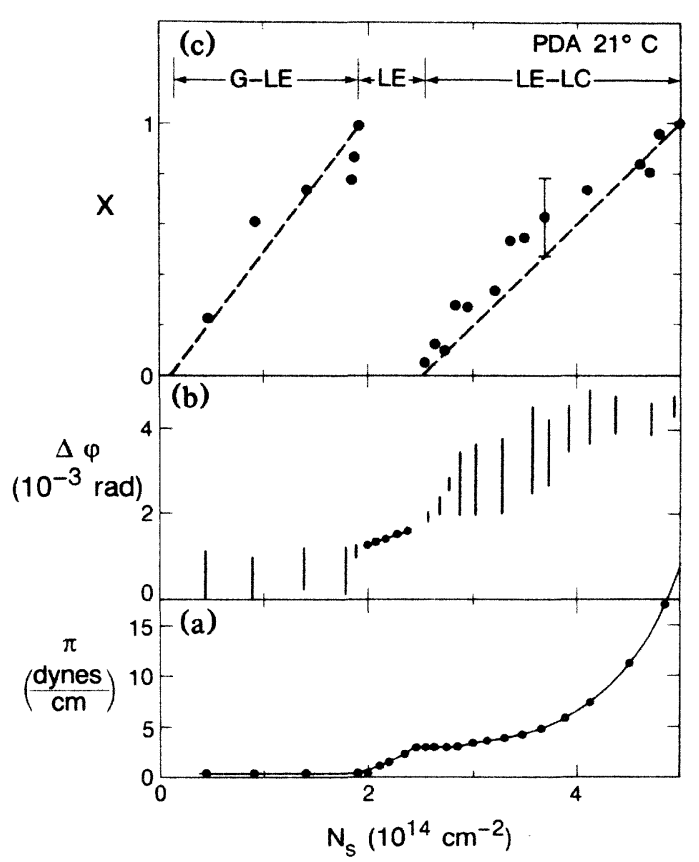

FIG. 2. (a) Surface pressure $\pi$, (b) observed phase shift $\Delta \phi$, and (c) domain fraction $x$ for a monolayer of PDA on water at $21^{\circ} \mathrm{C}$.

indicated by a kink in the pressure curve, the fluctuations appear again [see Fig. 1(c)]. When the density reaches a full monolayer at $N_{s}=N_{0}=5 \times 10^{14} \mathrm{~cm}^{-2}$, the amplitude of the fluctuations decreases but remains finite.

These results can be understood as follows. A firstorder phase transition, like the condensation of a liquid from the gas phase, is characterized by the appearance of high-density phase domains coexisting with the lowdensity phase. As a function of the average density, the domains will grow at the expense of the low-density phase until a full condensation has taken place. Consequently, the observed phase shift in such a coexistence region is expected to be

$$
\Delta \phi-<\Delta \phi<\Delta \phi_{+},
$$

where $\Delta \phi_{-(+)}$indicates the phase shift of the lower (higher) density phase. In the practical situation of a monolayer on a water substrate, the convective motion of the water will move the domains in and out of the fixed probe area causing the observed ellipsometry signal to fluctuate in time [see Figs. 1(a) and 1(c)]. The experimental results clearly indicate the existence of two coexistence regions on the isotherm, indicating a first-order character of both the G-LE and LE-LC transitions, in agreement with surface potential observations. ${ }^{11}$ Knowing the size of the probe area $\left(\sim 0.5 \mathrm{~mm}^{2}\right)$, an estimate of the domain size can be made by looking at the $\Delta \phi$ data IFigs. 1 and $2(b)$ ]. In the G-LE region, the signal fluctuates more or less stepwise even at very low $N_{s}$, i.e., $\Delta \phi$ is either at its maximum or minimum value. This indicates that this region of the phase diagram is characterized by a few, but quite large $\left(\gg 0.5 \mathrm{~mm}^{2}\right)$, domains. On the other hand, in the LE-LC region $\Delta \phi$ fluctuates much more rap- 
idly and with a relatively smaller amplitude indicating the presence of many but smaller domains $\left(<0.5 \mathrm{~mm}^{2}\right)$. (Note: the peak-to-peak amplitude in this region would vary from run to run.) In both cases, the domain sizes are much larger than those observed in lipid systems $\left(\mu \mathrm{m}^{2}\right)$ where one uses a dye fluorescence technique to make the domains visible. ${ }^{12}$ Electron microscopy pictures of PDA monolayers transferred onto a substrate in the LE-LC phase also show much smaller domain sizes. ${ }^{13}$

A quantitative analysis of the fluctuations can be given as follows. If $c(t)$ is the fraction of the domains in the probe area at time $t$, the time dependence of $\Delta \phi$ is given by

$$
\Delta \phi(t)=[1-c(t)] \Delta \phi_{-}+c(t) \Delta \phi_{+} .
$$

On the other hand, the fraction $x$ of domains at any intermediate density $N_{-} \leq N_{s} \leq N_{+}$is

$$
x=\left(N_{s}-N_{-}\right) /\left(N_{+}-N_{-}\right),
$$

where $N_{+(-)}$indicates the density of the high- (low-) density phase. Experimentally, $x$ can be obtained from a time average over an interval $T$ much larger than the fluctuation time:

$$
\begin{aligned}
x & =\frac{1}{T} \int_{0}^{T} c(t) d t \\
& =\frac{(1 / T) \int_{0}^{T} \Delta \phi(t) d t-\Delta \phi-}{\Delta \phi_{+}-\Delta \phi_{-}} .
\end{aligned}
$$

For the G-LE regions, $\Delta \phi$ - corresponds to the $G$ phase (i.e., $\left.\Delta \phi_{-} \equiv 0\right)$ and $\Delta \phi_{+}$to the value at the beginning of the LE phase. This is similar for the LE-LC region. However, here, because of the finite fluctuations at even a full monolayer $\left(N_{0}=5 \times 10^{14} \mathrm{~cm}^{-2}\right)$, we used $\Delta \phi_{+}$ $=\langle\Delta \phi(t)\rangle_{0}=$ time-averaged value at $N_{0}$. The results thus obtained are plotted in Fig. 1(c) and appear to agree very well with the calculated values for $x$ according to Eq. (9) (dashed lines).

As mentioned above, the ellipsometry signal still fluctuates at full monolayer coverage. However, by further compressing the molecules the fluctuations would disappear for $\pi>21.4 \mathrm{dyn} / \mathrm{cm}$ and $\Delta \phi$ would have a value anywhere within the range of the fluctuation amplitude at $N_{0}$. After stopping compression at $\pi=25 \mathrm{dyn} / \mathrm{cm}, \pi$ would drop with $\Delta \phi$ remaining constant. As soon as $\pi \leq 21.4$ $\mathrm{dyn} / \mathrm{cm}$, the fluctuations would reappear. This was completely reproducible, with only a possible change in the amplitude size.

These results show that the $\mathrm{LE}$ and $\mathrm{LC}$ phases are very different: whereas the LE domains merge into a homogeneous phase, the LC phase is characterized by an ensemble of domains that do not want to merge, i.e., they repel each other. This can simply be understood from the difference in structure of the two types of domains. The LC domains consist of closely packed aligned molecules ${ }^{6,7}$ giving rise to a net dipole moment that causes the domains to repel each other. ${ }^{14,15}$ This does not happen for the LE domains because of the random molecular orientation of this liquidlike phase. This repelling force will also cause the surface pressure to increase with increasing average density. At full coverage this repulsion can be overcome by overcompressing the monolayer ( $\pi \geq 21.4 \mathrm{dyn} / \mathrm{cm}$ at $21^{\circ} \mathrm{C}$ ) and "freezing" it in a given configuration. The ellipsometry signal will then depend on the domain fraction fixed in the probe area at that moment, which explains the experimental observation of a signal anywhere within the fluctuation amplitude. However, such an overcompressed film is not stable and, presumably, due to film collapse, the pressure will drop again and the fluctuations reappear.

Due to the fact that monolayer phase diagrams are usually measured by continuously compressing the monolayer while monitoring the surface pressure, this "freezing point" can easily be mistaken for another phase transition (LC $\rightarrow$ solid). Instead, from our observations we can conclude that the $\mathrm{LE} \rightarrow$ LC transition never does get totally completed as a result of the domain repulsion.

As mentioned earlier, the observed $\Delta \phi$ varies linearly with $N_{s}$ in the LE phase, in accordance with Eqs. (4) and (5). The same is true for the average behavior of $\Delta \phi-N_{s}$ in the G-LE and LE-LC regions [see Fig. 2(b)]. However, linear extrapolations of those data show that their slopes $S$ increase from the lower to the higher density regions: $S_{\mathrm{G}-\mathrm{LE}}<S_{\mathrm{LE}}<S_{\mathrm{LE}-\mathrm{LC}}$. This indicates that Eq. (5) is only approximately true: at higher densities, the molecular polarizabilities cannot simply be added due to the molecule-molecule interactions.

These local-field effects will also be reflected in the values for the indices of refraction $n_{m}=\sqrt{\epsilon_{m}}$ that can be calculated using Eq. (6). Because in this macroscopic approach $\Delta \phi$ is determined by $n_{m} d$, the thickness $d$ of the monolayer must be known. For the close-packed LC domains we take $d=d_{0}=18.8 \AA=$ length of a PDA molecule. ${ }^{16}$ From the maximum observed phase shift this yield $n_{\mathrm{LC}}=1.455 \pm 0.002$. For the LE phase, assuming an average thickness $d=d_{0} N_{s} / N_{0}$ one obtains $n_{\mathrm{LE}}=1.420$ \pm 0.005 . This shows that the LE $\rightarrow$ LC transition is accompanied by a small jump in the index of refraction of the monolayer. In addition, these results give an experimental justification for the macroscopic description of a monolayer as a thin dielectric sheet with a bulk index of refraction $n_{m}$ : the values found for $n_{m}$ are in good agreement with bulk values of similar hydrocarbons. ${ }^{17}$

In conclusion, by using a very sensitive ellipsometry setup together with a surface balance we have been able to study the appearance and average growth of domains in the G-LE and LE-LC coexistence regions for a monolayer of PDA on water. The G-LE region is characterized by fairly large $\left(\gg 0.5 \mathrm{~mm}^{2}\right)$ domains that merge into a homogeneous LE phase. In the LE-LC region, the domains are smaller and do not merge into a uniform phase, but rather form an aggregate. The first-order $\mathrm{LE} \rightarrow \mathrm{LC}$ transition is accompanied by a small jump in the index of refraction of the monolayer. The results do not support the distinction between a LC and solid phase.

This work was partially supported by the Director, Office of Energy Research, Office of Basic Energy Sciences, Materials Sciences Division of the U.S. Department of Energy under Contract No. DE-AC0376 SF00098. 
*Permanent address: Central Research and Development Department, E. I. du Pont de Nemours, Experimental Station, Wilmington, DE 19898.

${ }^{1}$ G. L. Larkins, E. D. Thompson, M. J. Deen, C. W. Burkhart, and J. B. Lando, IEEE Trans. Magn. 19, 980 (1983).

${ }^{2}$ M. C. Philips and D. Chapman, Biochim. Biophys. Acta 163, 301 (1986).

${ }^{3}$ G. M. Bell, L. L. Coombs, and J. L. Dunne, Chem. Rev. 81, 15 (1981).

${ }^{4}$ M. W. Kim and D. S. Cannell, Phys. Rev. Lett. 35, 889 (1975); Phys. Rev. A 13, 411 (1976).

${ }^{5}$ N. R. Pallas and B. A. Pethica, Langmuir 1, 509 (1985).

${ }^{6}$ Th. Rasing, Y. R. Shen, M. W. Kim, and S. Grubb, Phys. Rev. Lett. 55, 2903 (1985).

${ }^{7}$ P. Guyot-Sionnest, J. H. Hunt, and Y. R. Shen, Phys. Rev. Lett. 59, 1597 (1987).

${ }^{8}$ T. Smith, J. Opt. Soc. Am. 58, 1069 (1968).

${ }^{9}$ G. L. Gaines, Jr., Insoluble Monolayers at Liquid Gas Inter- faces (Wiley, New York, 1966).

${ }^{10}$ R. M. A. Azzam and N. M. Bashara, Ellipsometry and Polarized Light (North-Holland, Amsterdam, 1977).

${ }^{11} \mathrm{~S}$. R. Middleton and B. A. Pethica, Faraday Symp. Chem. Soc. 16, 109 (1981).

${ }^{12}$ H. M. McConnell, L. K. Tamm, and R. M. Weis, Proc. Natl. Acad. Sci. U.S.A. 81, 3249 (1984).

${ }^{13}$ S. Fereshtehkhou, R. D. Neuman, and R. Ovalle, J. Colloid Interface Sci. 109, 385 (1986).

${ }^{14}$ A. Fischer, M. Lösche, H. Möhwald, and E. Sackman, J. Phys. Lett. 45, L785 (1984).

${ }^{15}$ D. Andelman, F. Brochard, P. G. de Gennes, and J. F. Joanny, Acad. Sci. 301, 675 (1985).

${ }^{16}$ N. K. Adam Physics and Chemistry of Surfaces (Oxford, Univ. Press, London, 1946).

${ }^{17}$ CRC Handbook of Chemistry and Physics (CRS Press, Cleveland, 1974). 